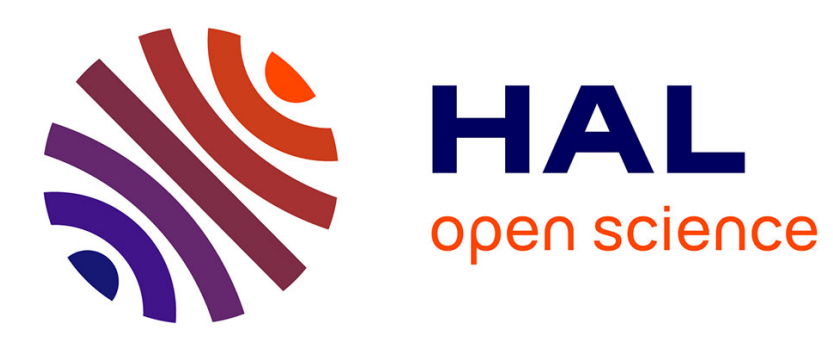

\title{
Aggregation of Heterogeneous Beliefs
}

\author{
Elyès Jouini, Clotilde Napp
}

\section{To cite this version:}

Elyès Jouini, Clotilde Napp. Aggregation of Heterogeneous Beliefs. Journal of Mathematical Economics, 2006, pp.752-770. halshs-00176505

\section{HAL Id: halshs-00176505 https://shs.hal.science/halshs-00176505}

Submitted on 4 Oct 2007

HAL is a multi-disciplinary open access archive for the deposit and dissemination of scientific research documents, whether they are published or not. The documents may come from teaching and research institutions in France or abroad, or from public or private research centers.
L'archive ouverte pluridisciplinaire HAL, est destinée au dépôt et à la diffusion de documents scientifiques de niveau recherche, publiés ou non, émanant des établissements d'enseignement et de recherche français ou étrangers, des laboratoires publics ou privés. 


\title{
Aggregation of heterogeneous beliefs*
}

\author{
E. Jouini \\ CEREMADE-Université Paris -Dauphine \\ and Institut universitaire de France \\ C. Napp \\ CEREG-Université Paris-Dauphine and CREST
}

July 7, 2006

\begin{abstract}
This paper is a generalization of Calvet et al. (2002) to a dynamic setting. We propose a method to aggregate heterogeneous individual probability beliefs, in dynamic and complete asset markets, into a single consensus probability belief. This consensus probability belief, if commonly shared by all investors, generates the same equilibrium prices as well as the same individual marginal valuation as in the original heterogeneous probability beliefs setting. As in Calvet et al. (2002), the construction stands on a fictitious adjustment of the market portfolio. The adjustment process reflects the aggregation bias due to the diversity of beliefs. In this setting, the construction of a representative agent is shown to be also valid.
\end{abstract}

\section{Introduction}

In a recent paper, Calvet, Grandmont and Lemaire (2002) propose a way to construct a representative agent in a static heterogeneous beliefs setting. In the present paper, we extend this approach to an intertemporal framework.

*The authors thank Jean-Michel Grandmont for helpful discussions and mainly for bringing this problem to their attention. The financial support of the Europlace Institute of Finance is gratefully acknowledged. 
The main purpose of Calvet et al. (2002) is to incorporate in the representation of the economy some degree of heterogeneity in the investors beliefs and to analyze how one could extend and modify the traditional "expected utility maximizing representative agent approach" in order to cover the case, which appears to be empirically more relevant, of heterogeneous beliefs. Among the issues the authors investigate are: is it possible to define a consensus probability that would aggregate heterogeneous individual subjective beliefs and could be used to explain (mimic) equilibrium prices? Is it still possible in such a context to define a version of an expected utility maximizing aggregate investor that would represent an equilibrium of this economy, i.e. generate the same equilibrium asset prices and mimic equilibrium pricing of assets by individuals? These issues are addressed in the simple framework of a static exchange economy. Given an observed equilibrium with heterogeneous individual subjective probabilities, Calvet et al. (2002) define an "equivalent equilibrium," where all investors would share the single consensus probability by: 1) the equivalent equilibrium generates the same equilibrium prices and 2) every investor's marginal expected utility valuations of an asset remain the same in both equilibria. They prove the existence of such an equivalent equilibrium modulo a scalar adjustment of the market portfolio. This means that in order to aggregate the individual heterogeneous beliefs into a single aggregate belief, the market portfolio may have to be scalarly adjusted, upward or downward, in the equivalent common probability equilibrium, a reflection of an "aggregation bias" due to the diversity of beliefs. Moreover, the authors show that the standard construction of an expected utility maximizing aggregate investor, who is designed so as to generate the observed equilibrium asset prices when endowed with the market portfolio, and to value then assets at the margin as does every individual investor in equilibrium, does carry over to the case of heterogeneous subjective probabilities, provided that 1) this aggregate investor is assigned the same consensus probability as previously found and that 2 ) the market portfolio (aggregate consumption) is scalarly adjusted upwardly or downwardly as previously.

The aim of the present paper is to address the same issues as Calvet et al. (2002) but in an intertemporal, discrete or continous time, framework. We start in Section 2 by introducing our dynamic and complete markets model. Given an observed heterogeneous beliefs Arrow-Debreu equilibrium, we then define an equivalent equilibrium where all investors would share a common "consensus belief" by the same requirements as in Calvet et al. (2002), i.e. invariance of the equilibrium prices and of the investors marginal valuations. We prove (Subsec- 
tion 2.1) that in a dynamic setting a scalar adjustment of the market portfolio is not sufficient in order to ensure the existence of an equivalent equilibrium. We introduce a predictable adjustment of the market portfolio and prove the existence (and unicity) of an equivalent equilibrium under this condition (Subsection 2.2). This means that equilibrium prices and individual marginal valuations in the heterogeneous beliefs setting are the same as in an otherwise similar homogeneous beliefs economy in which aggregate endowment is modified, upward or downward, in a predictable way. We also show that this homogeneous beliefs equivalent equilibrium is naturally associated to a representative agent endowed with the adjusted market portfolio (Subsection 2.3). We end Section 2 by showing on specific examples (HARA utility functions) how the consensus probability and the predictable adjustment process can be explicitly obtained. We find that the consensus probability is directly related to some weighted mean of individual subjective beliefs. We also characterize the situations where the adjustment of the market portfolio has to be made upward (resp. downward). In particular, we find that the adjustment process is nondecreasing and greater than 1 (resp. nonincreasing and smaller than 1) hence leads to an equivalent equilibrium in which aggregate endowment is increased (resp. decreased) if the cautiousness parameter is smaller than 1 (resp. greater than 1). In a standard setting without beliefs heterogeneity, when there is more risk involved and when the investor is cautious (i.e. its cautiousness parameter is smaller than 1), it can be shown that the investor will increase current consumption acting as if future wealth was decreased. Now in our context, a possible interpretation of our result consists in considering the dispersion of beliefs as a source of risk, thereby leading for the representative agent to an upward or downward adjustment of aggregate endowment depending on whether the cautiousness parameter is smaller or greater than 1. Our results are consistent with those of Rubinstein (1974) and Zapatero (1998) where the only effect of the heterogeneous beliefs on the asset prices is related to a change of probability (no scaling effect). Indeed, in these papers, the agents are endowed with logarithmic utility functions and it appears that these functions are the only ones in the HARA class for which the predictable adjustment process is constant and equal to 1 .

Section 3 essentially consists of remarks and extensions. We first explore the implications of the aggregation procedure on the risk sharing rule. In the standard case of homogeneous beliefs and state independent utilities, we know that the equilibrium allocations are comonotonic, i.e. are increasing functions of the same factor (the aggregate consumption) and this feature is commonly called the 
risk sharing rule. We show that our results can be reinterpreted as generalizing the standard risk sharing rule result to the case of heterogeneous beliefs. Indeed, as in Calvet et al. (2002), we show that, in a dynamic framework, the equilibrium allocations in an heterogeneous beliefs setting can be divided into two parts. The first part satisfies the standard risk sharing rule and the second part can be interpreted as a residual risk due to heterogeneity of beliefs and is monotone in individual beliefs deviations from the consensus probability.

We also show (Subsection 3.2) how the same issues can be addressed in a continuous time setting. We prove in such a context the existence of an equivalent equilibrium satisfying the same requirements as before, modulo an adjustment of the market portfolio, which is a finite variation process.

In Subsection 3.3, we analyze the second invariance requirement of our equivalent equilibrium, to wit, that the investors marginal utilities remain the same in both equilibria, and more precisely its links with the desirable property for an equivalent equilibrium that each investor's observed (or initial) demand be larger than (resp. equal to, less than) his demand in the equivalent equilibrium if and only if he attaches a subjective probability that is larger than (resp. equal to, less than) the consensus probability.

Finally, we consider in Subsection 3.4 alternative aggregation procedures.

All the proofs are in the Appendix.

\section{Aggregation of heterogeneous beliefs in discrete time}

We consider a collection of individual investors indexed by $i=1, \ldots, N$. We fix a finite time horizon $T$ on which we are going to treat our problem and we consider a filtered complete probability space $\left(\Omega, F,\left(F_{t}\right)_{t=0}^{T}, P\right)$, which satisfies the usual conditions ${ }^{1}$. The set $\Omega$ represents the set of all histories and the $\sigma$-algebra $F_{t}$ can be thought of as representing all (theoretically) observable events up to and including time $t$. Each investor solves a standard dynamic utility maximization problem. He has a current endowment at date $t$ denoted by $e_{t}^{*^{i}}$, and a Von Neumann Morgenstern utility function for consumption of the form $E\left[\sum_{t=0}^{T} M_{t}^{i} u_{i}\left(t, c_{t}\right)\right]$, where $M_{t}^{i}$ is a positive martingale process satisfying $M_{0}^{i}=1$, i.e. the positive den-

\footnotetext{
${ }^{1}$ A filtered complete probability space $\left(\Omega, F,\left(F_{t}\right)_{t=0}^{T}, P\right)$ is said to satisfy the usual conditions if (i) $F_{0}$ contains all the $P$-null sets of $F$ and (ii) $F_{t}=\cap_{u>t} F_{u}$, all $t, 0 \leq t<\infty$, i.e., the filtration is right-continuous.
} 
sity process of a probability measure $Q^{i}$ equivalent to $P$, and corresponds to the subjective belief of individual $i$. We make the following assumptions

1. For all $t=0, \ldots, T, u_{i}(t, \cdot): \mathbb{R}_{+} \rightarrow \mathbb{R} \cup\{-\infty\}$ is of class $C^{1}$ on $\mathbb{R}_{+}^{*}$, strictly increasing and strictly concave.

2. For all $t, u_{i}(t, \cdot)$ satisfies Inada conditions, i.e. the derivative of $u_{i}(t, \cdot)$ denoted by $u_{i}^{\prime}(t, \cdot)$ is such that $u_{i}^{\prime}\left(t, 0_{+}\right)=\infty$ and $u_{i}^{\prime}(t, \infty)=0$. We shall denote by $\left(u_{i}^{\prime}\right)^{-1}(t, \cdot)$ the inverse function of $u_{i}^{\prime}(t, \cdot)$, which is continuous and strictly decreasing.

3. The aggregate endowment $e^{*} \equiv \sum_{i=1}^{N} e^{*^{i}}$ satisfies $\underline{e} \leq e_{t}^{*} \leq \bar{e}$ uniformly in $(t, \omega)$ for some positive constants $\underline{e}$ and $\bar{e}$.

We recall that an Arrow-Debreu equilibrium relatively to the beliefs $\left(M^{i}\right)$ and the endowment processes $\left(e^{i}\right)$ is defined by a positive, uniformly bounded price process $q^{*}$ and a family of optimal admissible consumption plans $\left(y^{*^{i}}\right)$ such that markets clear, i.e.

$$
\left\{\begin{array}{c}
y^{*^{i}}=y^{i}\left(q^{*}, M^{i}, e^{i}\right) \\
\sum_{i=1}^{N} y^{*^{i}}=\sum_{i=1}^{N} e^{i} \equiv e^{*}
\end{array}\right.
$$

where

$$
y^{i}(q, M, e) \equiv \arg \max _{E^{P}\left[\sum_{t=0}^{T} q_{t}\left(y_{t}^{i}-e_{t}\right)\right] \leq 0} E^{P}\left[\sum_{t=0}^{T} M_{t} u_{i}\left(c_{t}\right)\right] .
$$

We start from an Arrow-Debreu equilibrium $\left(q^{*},\left(y^{*^{i}}\right)\right)$ relatively to the beliefs $\left(M^{i}\right)$ and the endowment processes $e^{i}$. Such an equilibrium, when it exists, can be characterized by the first order necessary conditions for individual optimality and the market clearing condition. These conditions can be written as follows

$$
\left\{\begin{aligned}
M_{t}^{i} u_{i}^{\prime}\left(t, y^{*^{i}}\right) & =\lambda_{i} q_{t}^{*}, \\
E^{P}\left[\sum_{t=0}^{T} q_{t}^{*}\left(y_{t}^{*^{i}}-e_{t}^{i}\right)\right] & =0 \quad i=1, \ldots, N \\
\sum_{i=1}^{N} y^{*^{2}} & =e^{*}
\end{aligned}\right.
$$

for some set of positive Lagrange multipliers $\left(\lambda_{i}\right)$.

Our aim is to find an "equivalent equilibrium" in which the heterogeneous subjective beliefs would be aggregated into a common belief $M$, i.e. all investors would share the common belief $M$, the process $M$ being like the $M^{i}$ 's a positive 
martingale process satisfying $E\left[M_{T}\right]=1$, i.e. the positive density of a probability measure $Q$ equivalent to $P$. We want the "equivalent equilibrium" to generate the same equilibrium price process $q^{*}$ as in the original equilibrium with heterogeneous beliefs, so that every asset gets the same valuation in both equilibria. The first order conditions would then lead to

$$
M_{t} u_{i}^{\prime}\left(t,{\overline{y_{t}}}^{i}\right)=\alpha_{i} M_{t}^{i} u_{i}^{\prime}\left(t, y_{t}^{*^{i}}\right)
$$

where ${\overline{y_{t}}}^{i}$ stands for the new equilibrium allocation and where $\alpha_{i}$ is a given positive multiplier. Taking the product of all these equations in the particular case where $u_{i}^{\prime}(t, x)=\exp -(x / \theta)$, we obtain

$$
M_{t}=\left(\prod_{i=1}^{N} \alpha_{i}\right)^{1 / N}\left(\prod_{i=1}^{N} M_{t}^{i}\right)^{1 / N}
$$

In that case, it appears that $M$ is a martingale only if all the $M_{i}$ 's are equal. There is therefore in general no solution to the aggregation problem. Calvet et al. (2002) in a static framework propose to permit a scalar adjustment of the aggregate endowment and to replace the invariance principle on the aggregate endowment by an invariance principle on the individual marginal valuation of assets.

\subsection{Aggregation of heterogeneous beliefs with a scalar adjustment of the market portfolio}

Following the approach of Calvet et al. (2002), we define the "equivalent equilibrium" by two requirements. First, the "equivalent equilibrium" should generate the same equilibrium price process $q^{*}$ as in the original equilibrium with heterogeneous beliefs, so that every asset gets the same valuation in both equilibria. Second, every investor should be indifferent at the margin between investing one additional unit of income in the original equilibrium with heterogeneous beliefs and in the "equivalent equilibrium", so that every asset gets the same marginal valuation by each investor (in terms of his marginal expected utility) in both equilibria. We show in Section 3 that this requirement is essentially equivalent to a monotonicity requirement between changes in the individual portfolios and the underlying changes of individual probabilities.

Calvet et al. (2002) prove in a static setting that the construction of an homogeneous beliefs equivalent equilibrium designed so as to mimic equilibrium 
prices and marginal asset valuations by individual investors, is possible in a diverse beliefs framework. The proposed design may require a scalar adjustment of the market portfolio.

We show on the following example that this result does not extend to the dynamic setting, i.e., prices and individual marginal valuations in an heterogeneous probability beliefs setting cannot necessarily be expressed as prices and individual marginal valuations in an homogeneous probability beliefs economy with a possible scalar adjustment on aggregate endowment.

Let $\Omega \equiv\left\{\omega_{1}, \omega_{2}\right\}, P=(1 / 2,1 / 2), M^{1} \equiv 1$ and $M^{2}$ be such that

$$
M_{0}^{2}=1, M_{1}^{2}\left(\omega_{1}\right)=2 / 3, M_{1}^{2}\left(\omega_{2}\right)=4 / 3 .
$$

Let $u(\cdot)=u_{1}(t, \cdot)=u_{2}(t, \cdot)$ be such that $u^{\prime}(x)=x^{\alpha}$ with $\alpha \in[-1,0[$. We take $e^{*^{i}}$ such that $M^{i} u^{\prime}\left(e^{*^{i}}\right)$ does not depend upon $i$ and we fix $q^{*} \equiv M^{1} u^{\prime}\left(e^{*^{1}}\right)$. In this setting, $\left(q^{*},\left(e^{*^{i}}\right)\right)$ is an equilibrium relative to the beliefs $\left(M^{i}\right)$ and the endowment processes $e^{*^{i}}$. We look for $\bar{M}, \bar{y}^{1}, \bar{y}^{2}$ and $\bar{r} \in \mathbb{R}$ such that for $i=1,2$, we have

$$
\begin{aligned}
\bar{M} u^{\prime}\left(\bar{y}^{i}\right) & =M^{i} u^{\prime}\left(e^{*^{i}}\right) \quad i=1,2 \\
\sum_{i} \bar{y}^{i} & =\bar{r} \sum_{i} e^{*^{i}}
\end{aligned}
$$

It appears that $\frac{1 / 2 \bar{M}_{1}\left(\omega_{1}\right)+1 / 2 \bar{M}_{1}\left(\omega_{2}\right)}{\bar{M}_{0}}$ is given by $2\left[\frac{1 / 3}{\left(1+(2 / 3)^{1 / \alpha}\right)^{\alpha}}+\frac{2 / 3}{\left(1+(4 / 3)^{1 / \alpha}\right)^{\alpha}}\right]^{1 / \alpha}$, which is equal to one if and only if $\alpha=-1$. Consequently, except for the logarithmic utility function, all the other utility functions in our class lead to processes $\bar{M}$ that are not martingales. Hence, they can not be interpreted as the density process of a given probability.

\subsection{Aggregation of heterogeneous beliefs into a consensus probability belief and a predictable adjustment process of the market portfolio}

We have just seen in the previous subsection that we cannot in general aggregate the beliefs $M^{i}$ in such a way that $M$ is a martingale while keeping the adjustment of the market portfolio a scalar. We shall introduce a predictable adjustment process. We shall indifferently use the terminology "equilibrium relative to the beliefs $Q^{i}$ " or "equilibrium relative to the beliefs $M^{i}$," where as above $M^{i}$ represents the density process of $Q^{i}$ with respect to $P$. 
Proposition 2.1. Consider an Arrow-Debreu equilibrium $\left(q^{*},\left(y^{*^{i}}\right)\right)$ relative to the beliefs $\left(Q^{i}\right)$ and the endowment processes $e^{i}$ with $\sum_{i=1}^{N} e^{i} \equiv e^{*}$. There exists a unique equivalent probability measure $\bar{Q}$, a unique positive and bounded predictable adjustment process $\bar{r}$, a unique family of endowment processes $\left(\bar{e}^{i}\right)$ with $\sum_{i=1}^{N} \bar{e}^{i}=\bar{r} e^{*}$ and a unique family of consumption processes $\left(\bar{y}^{i}\right)$ such that

1. $\left(q^{*},\left(\bar{y}^{i}\right)\right)$ is an equilibrium relative to the common belief $\bar{Q}$ and the endowment processes $\left(\bar{e}^{i}\right)$

2. Trading volumes and individual marginal valuation remain the same before and after the aggregation procedure, i.e. for all $i=1, \ldots, N$

$$
y^{*^{i}}-e^{i}=\bar{y}^{i}-\bar{e}^{i}
$$

and

$$
u_{i}^{\prime}\left(t, y_{t}^{*^{i}}\right)=u_{i}^{\prime}\left(t, \bar{y}^{i}\right)
$$

Notice that this aggregation procedure satisfies the additional homogeneity requirement. We obtain through this aggregation procedure that equilibrium prices and individual marginal valuations in the heterogeneous beliefs setting are the same as in an otherwise similar homogeneous beliefs economy in which aggregate endowment is adjusted in a predictable way. A natural question is then to determine whether this adjustment of the market portfolio is to be made upward or downward. We shall at the end of Section 2 analyze, in the specific case of HARA utility functions, the situations leading to an upward (resp. downward) adjustment of the market portfolio. We start by showing in the next section that this homogeneous beliefs equivalent equilibrium is naturally associated to a representative agent endowed with the adjusted market portfolio (Subsection 2.3).

\subsection{Representative agent}

As in the standard case of homogeneous beliefs, we want to construct an expected utility maximizing aggregate investor, representing the economy in equilibrium. More precisely, we look for a single aggregate investor, endowed with the market portfolio, who, when maximizing his expected utility under the consensus probability generates the same equilibrium prices as in the original equilibrium. The next proposition establishes the existence of such a representative agent, as long 
as the market portfolio is being adjusted by the same predictable process $\bar{r}$ as before. As in the standard case, for $\alpha \in\left(\mathbb{R}_{+}^{*}\right)^{N}$, we introduce the function

$$
u_{\alpha}(t, x)=\max _{\sum_{i=1}^{N} x_{i} \leq x} \sum_{i=1}^{N} \frac{1}{\alpha_{i}} u_{i}\left(t, x_{i}\right)
$$

and we recall that we denote by $\lambda \in\left(\mathbb{R}_{+}^{*}\right)^{N}$ the positive Lagrange multipliers of the heterogeneous beliefs initial equilibrium.

Proposition 2.2. Consider an equilibrium $\left(q^{*},\left(y^{*^{i}}\right)\right)$ relative to the beliefs $\left(Q^{i}\right)$ and the endowment processes $e^{i}$ with $\sum_{i=1}^{N} e^{i} \equiv e^{*}$. The aggregate investor defined by the normalized VNM utility $u_{\lambda}$ is an equilibrium representative agent when endowed with the common probability $\bar{Q}$ as in Proposition 2.1, and the adjusted market portfolio $\bar{r} e^{*}$, in the sense that the portfolio $\bar{r} e^{*}$ maximizes his expected utility $E^{\bar{Q}}\left[\sum_{t=0}^{T} u_{\lambda}\left(t, y_{t}\right)\right]$ under the market budget constraint $E\left[\sum_{t=0}^{T} q_{t}^{*}\left(y_{t}-\bar{r}_{t} e_{t}^{*}\right)\right] \leq$ 0 .

The construction of the representative agent is exactly the same as in the standard setting. As a consequence, all classical properties of the representative agent utility function remain valid in our setting (see e.g. Huang-Litzenberger, 1988). Among other properties, if all individual utility functions are state independent, then the aggregate utility function is also state independent, and if all individual utility functions exhibit linear risk tolerance, i.e. are such that $-\frac{u_{i}^{\prime}(t, x)}{u_{i}^{\prime \prime}(t, x)}=\theta_{i}+\eta x$, then the aggregate utility function is also such that $-\frac{u^{\prime}(t, x)}{u^{\prime \prime}(t, x)}=\bar{\theta}+\eta x$ where $\bar{\theta}=\sum_{i=1}^{N} \theta_{i}$.

Remark that our aggregation procedure applies to a framework where agents have common beliefs but possibly different state dependent utility functions of the following "separable" form

$$
U_{i}(t, \omega, x)=v_{i}(t, \omega) u_{i}(t, x) .
$$

In that case ${ }^{2}$, we obtain a representative agent utility function of the same form $U(t, \omega, x)=v(t, \omega) u(t, x)$, where the function $u$ is obtained from the $u_{i}$ 's as in the standard framework, and where $v$ is an average of the $v_{i}$ 's.

\footnotetext{
${ }^{2}$ Note that even if the $v_{i}$ 's are not martingales, our results still apply.
} 
Our construction can be compared with Cuoco-He (1994) representative agent construction in incomplete market models (or Basak-Cuoco (1998) in models with restricted market participation). The main difference is that in Cuoco and He's construction, the representative agent 's utility function appears as a stochastic weighted average of the individual utility functions, whereas in our construction, the weights are deterministic, the representative agent's utility function is the same as in the standard case, however the total endowment is stochastically adjusted. In particular, as underlined above, in our construction, the classical properties of the representative agent's utility function remain valid, which is not the case in Cuoco and He's construction..Besides, our construction (or more precisely, Calvet et al. 's contruction) seems to be more tractable in order to compare the equilibrium characteristics in the standard and in the heterogeneous beliefs settings.

\subsection{Example: HARA utility functions}

In this subsection we assume that all the utility functions are in the HARA class. More precisely, we suppose that $u_{i}(t, x)$ is such that $-\frac{u_{i}^{\prime}(t, x)}{u_{i}{ }^{\prime \prime}(t, x)}=\theta_{i}+\eta x$ for all $i$. Although some of these functions do not satisfy Inada conditions, the consensus probability and the predictable adjustment process, if they exist, should satisfy the same first order conditions as before and any pair $(M, r)$ satisfying these conditions solves our aggregation problem. We obtain in the next proposition as in Calvet et al. (2002) explicit expressions for the agggregate consensus probability and the adjustment process $\bar{r}$ in the case of HARA utility functions. In particular, we are able to determine if the aggregation bias contributes to an "increase" or a "decrease" of aggregate endowment.

Proposition 2.3. Assume that individual VNM utilities belong to the HARA family with $-\frac{u_{i}^{\prime}(t, x)}{u_{i}{ }^{\prime}(t, x)}=\theta_{i}+\eta x>0$.

1. The representative agent who supports the equilibrium with the common probability $\bar{Q}$ in Proposition 2.2 belongs to the same HARA family with $-\frac{u_{\lambda}^{\prime}(t, x)}{u_{\lambda}{ }^{\prime}(t, x)}=\bar{\theta}+\eta x$, where $\bar{\theta}=\sum_{i=1}^{N} \theta_{i}$.

2. The density $\bar{M}$ with respect to $P$ of the corresponding common probability $\bar{Q}$ and the adjustment process $\bar{r}$ are given 
- when $\eta \neq 0$, by

$$
\begin{aligned}
& \bar{M} \text { is a martingale } \\
\bar{r}_{0}= & M_{0}=1 \\
\bar{M}_{t}= & \left(\frac{\bar{\theta}+\eta \bar{r}_{t} e_{t}^{*}}{\bar{\theta}+\eta e_{t}^{*}}\right)^{1 / \eta}\left[\sum_{i=1}^{N} \gamma_{i}\left(M_{t}^{i}\right)^{\eta}\right]^{1 / \eta}
\end{aligned}
$$

with $\gamma_{i}=\frac{\lambda_{i}^{-\eta}}{\sum_{i=1}^{N} \lambda_{i}^{-\eta}}$.

- when $\eta=0$, by

$$
\begin{aligned}
\bar{M} \text { is a martingale } \\
\bar{r}_{0}=\overline{M_{0}}=1 \\
\overline{M_{t}}=\prod_{i=1}^{N}\left(M_{t}^{i}\right)^{\theta_{i} / \bar{\theta}} \exp \frac{\left(\overline{r_{t}}-1\right) e_{t}^{*}}{\bar{\theta}}
\end{aligned}
$$

3. The adjustment process $\bar{r}$ satisfies

$$
\begin{aligned}
\bar{r}(t, \omega) \geq 1 \text { if } \eta & <1 \\
\bar{r}(t, \omega) \leq 1 \text { if } \eta & >1 \\
\bar{r}(t, \omega) & =1 \text { if } \eta=1
\end{aligned}
$$

4. If $\bar{\theta} \equiv \sum_{i=1}^{N} \theta_{i}=0$, then we obtain a simple construction algorithm

$$
\begin{aligned}
\bar{r}_{0} & =\overline{M_{0}}=1 \\
\bar{r}_{t} & =\frac{\bar{M}_{t-1}^{\eta}}{E_{t-1}\left[\left(\sum_{i=1}^{N} \gamma_{i}\left(M_{t}^{i}\right)^{\eta}\right)^{1 / \eta}\right]} \\
\left(\overline{M_{t}}\right)^{\eta} & =\overline{r_{t}}\left[\sum_{i=1}^{N} \gamma_{i}\left(M_{t}^{i}\right)^{\eta}\right]
\end{aligned}
$$

and the predictable adjustment process $\bar{r}$ satisfies

$$
\begin{aligned}
& \bar{r} \text { is nondecreasing if } \eta<1 \\
& \bar{r} \text { is nonincreasing if } \eta>1
\end{aligned}
$$


Notice that the consensus belief is always given by some weighted mean of the individual heterogeneous beliefs, adjusted by a process depending on $\bar{r}$. The mean is either a geometric mean (in the case of exponential utility functions) or a "power- $\eta$ " mean (in the case of power utility functions). The weights are given by the individual risk tolerances $T_{i} \equiv \frac{\theta_{i}+\eta y^{*^{*}}}{\bar{\theta}+\eta e^{*}}$. The process $\bar{r}$ is directly related to the dispersion of the beliefs $M^{i}$.

In the specific case of power utility functions with $\theta_{i}=0$, i.e. if for all $i=$ $1, \ldots, N, u_{i}^{\prime}(t, x)=(\eta x)^{-1 / \eta}$, then

$$
\bar{M}_{t}=\left(\overline{r_{t}}\right)^{1 / \eta}\left[\sum_{i=1}^{N} \gamma_{i}\left(M_{t}^{i}\right)^{\eta}\right]^{1 / \eta} .
$$

The process $\bar{r}$ measures the default of martingality of the mean $\left[\sum_{i=1}^{N} \gamma_{i}\left(M_{t}^{i}\right)^{\eta}\right]^{1 / \eta}$. We know by the previous proposition that $\bar{r}$ is nondecreasing (resp. nonincreasing) if $\eta<1$ (resp. $\eta>1$ ). The interpretation is the following. In the standard setting, when there is more risk involved, depending on whether the investor is cautious or not, that is to say depending on whether the cautiousness parameter is smaller or greater than 1, it can be shown that the investor will increase or reduce current consumption acting as if future wealth was decreased or increased. For instance, a cautious investor (cautiousness parameter smaller than 1) increases current consumption acting as if future wealth was increased. Now in our context with heterogeneous beliefs, a possible interpretation consists in considering the dispersion of beliefs as a source of risk, thereby leading for the representative agent to an upward or downward adjustment of aggregate endowment depending on whether the cautiousness parameter is smaller or greater than 1 .

In particular if all agents have the same logarithmic utility functions, i.e. if for all $i=1, \ldots, N, u_{i}^{\prime}(t, x)=x^{-1}$, then $\bar{r} \equiv 1$ and

$$
\bar{M}_{t}=\sum_{i=1}^{N} \gamma_{i} M_{t}^{i}
$$

where $\gamma_{i}=\frac{\lambda_{i}^{-1}}{\sum_{i=1}^{N} \lambda_{i}^{-1}}=\frac{y_{0}^{*^{i}}}{\sum_{i=1}^{N} y_{0}^{*}}$. In this case, we find that there is no adjustment effect on the market portfolio, and the consensus belief is given by a weighted arithmetic mean of the individual heterogeneous beliefs. This is the result of Rubinstein (1976). 


\section{Remarks and extensions}

\subsection{Risk sharing rule}

As underlined by Rubinstein (1976), one potential use of the aggregation procedure is to relate the heterogeneity of individual demands to the heterogeneity of individual beliefs. It is well known that in an homogeneous beliefs setting all the individual allocations are comonotonic. This property is called "the risk sharing rule." Let us see in our setting the implications of the aggregation procedure in terms of risk sharing rule. In the "equivalent equilibrium" $\left(q^{*}, \bar{Q}, \bar{r} e^{*}, \overline{e^{i}}\right)$, the beliefs are homogeneous (represented by the probability measure $\bar{Q}$ ) and all the individual allocations $\bar{y}^{i}$ are then comonotonic. In the initial equilibrium with heterogeneous beliefs, the individual allocations at time $t$ denoted by $y_{t}^{*^{i}}$ are given by

$$
\begin{aligned}
y_{t}^{*^{i}} & =\bar{y}_{t}^{i}+\left(y_{t}^{*^{i}}-\bar{y}_{t}^{i}\right) \\
& =\bar{y}_{t}^{i}+\varphi_{t}^{i}\left(M_{t}^{i}, \bar{M}_{t}\right)
\end{aligned}
$$

where for all $t$, the $\bar{y}_{t}^{i}$,s are comonotonic and $\varphi_{t}^{i}\left(M_{t}^{i}, \bar{M}_{t}\right) \geq 0$ when $M_{t}^{i} \geq \bar{M}_{t}$, and $\varphi_{t}^{i}\left(M_{t}^{i}, \bar{M}_{t}\right) \leq 0$ when $M_{t}^{i} \leq \bar{M}_{t}$. Furthermore $\varphi_{t}^{i}\left(M_{t}^{i}, \bar{M}_{t}\right)=\left(u_{i}^{\prime}\right)^{-1}\left(\frac{\bar{M}_{t}}{M_{t}^{i}} u_{i}^{\prime}\left(\bar{y}_{t}^{i}\right)\right)-$ $\bar{y}_{t}^{i}$ is monotone with respect to $M_{t}^{i}$. The heterogeneity of the beliefs induces then a distortion of the risk sharing rule and, for each agent, this distortion is monotone in individual beliefs deviations from the aggregate probability.

The function $\varphi_{t}^{i}$ can be explicitly computed for some specific classes of utility functions. For instance, in the exponential case, we get that

$$
y_{t}^{*^{i}}-\bar{y}_{t}^{i}=\theta_{i}\left(\log M_{t}^{i}-\log \bar{M}_{t}\right)
$$

\subsection{Aggregation of heterogeneous beliefs in a Continuous Time Setting}

Let us now consider a continuous time framework. We fix a finite time horizon $T$ on which we are going to treat our problem, we let $\mathbb{T} \equiv[0, T]$, and we consider a filtered probability space $\left(\Omega, F,\left(F_{t}\right)_{t=0}^{T}, P\right)$, where $\left(F_{t}\right)_{t \in \mathbb{T}}$ denotes the $P$ -augmentation of the natural filtration generated by a Brownian motion $W$ on $(\Omega, F, P)$. We assume that $F_{T}=F$. 
The total endowment of the economy is described by a stochastic process $e^{*}$ satisfying the following stochastic differential equation

$$
d e_{t}^{*}=\alpha_{t} e_{t}^{*} d t+\beta_{t} e_{t}^{*} d W_{t}
$$

and $e_{0}^{*}$ is given.

As previously, we assume that each individual's subjective belief $M^{i}$ is given by the positive density process of a probability measure $Q^{i}$, which is equivalent to the original probability measure $P$. Such a positive density process can be represented as the solution of the following stochastic differential equation

$$
d M_{t}^{i}=\delta_{t}^{i} M_{t}^{i} d W_{t}, \quad M_{0}^{i}=1
$$

We recall that an Arrow-Debreu equilibrium relative to the beliefs $\left(M^{i}\right)$ and the endowment processes $\left(e^{i}\right)$ is defined by a positive, uniformly bounded price process $q^{*}$ and a family of optimal admissible consumption plans $\left(y^{*^{i}}\right)$ such that markets clear, i.e.

$$
\left\{\begin{array}{c}
y^{*^{i}}=y^{i}\left(q^{*}, M^{i}, e^{i}\right) \\
\sum_{i=1}^{N} y^{*^{i}}=\sum_{i=1}^{N} e^{i} \equiv e^{*}
\end{array}\right.
$$

where

$$
y^{i}(q, M, e) \equiv \arg \max _{E^{P}\left[\int_{0}^{T} q_{t}\left(y_{t}^{i}-e_{t}\right) d t\right] \leq 0} E^{P}\left[\int_{0}^{T} M_{t} u_{i}\left(c_{t}\right) d t\right] .
$$

We start from an Arrow-Debreu equilibrium $\left(q^{*},\left(y^{*^{i}}\right)\right)$ relative to the beliefs $\left(M^{i}\right)$ and the endowment processes $e^{i}$ and we assume that the equilibrium allocations $\left(y^{*^{i}}\right)$ satisfy the following equations

$$
d y_{t}^{*^{i}}=\alpha_{t}^{i} y_{t}^{*^{i}} d t+\beta_{t}^{i} y_{t}^{*^{i}} d W_{t}
$$

The $\mathbb{R}$-valued processes $\left\{\alpha_{t} ; t \in \mathbb{T}\right\},\left\{\alpha_{t}^{i} ; t \in \mathbb{T}\right\},\left\{\beta_{t} ; t \in \mathbb{T}\right\},\left\{\beta_{t}^{i} ; t \in \mathbb{T}\right\}$, $\left\{\delta_{t}^{i} ; t \in \mathbb{T}\right\}$ are the coefficients of the model and are taken to be progressively measurable with respect to $\left(F_{t}\right)_{t \in \mathbb{T}}$ and bounded uniformly in $(t, \omega)$ in $\mathbb{T} \times \Omega$.

Furthermore, we clearly have

$$
\left\{\begin{array}{l}
\sum_{i=1}^{N} \alpha^{i} y^{*^{i}}=\alpha e^{*} \\
\sum_{i=1}^{N} \beta^{i} y^{*^{i}}=\beta e^{*}
\end{array}\right.
$$


Recall that our conditions on the density $\bar{M}$ and on the "equivalent equilibrium" lead to find an adjustment process $\bar{r}$, a density $\bar{M}$ and an allocation scheme $\left(\bar{y}^{i}\right)$ such that

$$
\sum_{i=1}^{N} \bar{y}^{i}=\bar{r} e^{*}
$$

and

$$
M^{i} u_{i}^{\prime}\left(\cdot, y^{*^{i}}\right)=\bar{M} u_{i}^{\prime}\left(\cdot, \bar{y}^{i}\right)
$$

for all $i$. We have seen that we can not impose that $\bar{r}$ be a scalar if we want $\bar{M}$ to be the density process of a probability measure equivalent to the initial probability measure $P$, i.e. a positive martingale with $M_{0}=1$. In the previous sections, we imposed that $\bar{r}$ be a positive predictable process and the uniqueness of such an $\bar{r}$ confirms that this condition is a natural condition. In the present setting, we are dealing with continuous processes and there is no difference between predictable processes and adapted processes. The predictability condition becomes then too weak. Indeed, it suffices to take any $\bar{M}$, to choose the $\bar{y}^{i}$ 's accordingly, i.e. such that $M^{i} u_{i}^{\prime}\left(\cdot, y^{*^{i}}\right)=\bar{M} u_{i}^{\prime}\left(\cdot, \bar{y}^{i}\right)$ and finally to take $\bar{r}=\sum_{i=1}^{N} \bar{y}^{i} / e^{*}$. The right condition in the current setting seems to be a zero-diffusion coefficient for $\bar{r}$, i.e.

$$
d \bar{r}_{t}=\rho_{t} \bar{r}_{t} d t
$$

for some process $\left(\rho_{t}\right)$.

In the next, we assume that the utility functions are $C^{1,3}$. If we impose that the $\bar{y}^{i}$ s and $\bar{M}$ be solutions of stochastic differential equations of the form

$$
\begin{aligned}
d \bar{y}_{t}^{i} & =a_{t}^{i} \overline{\bar{y}}^{i} d t+b_{t}^{i} \bar{y}_{t}^{i} d W_{t} \\
d \bar{M}_{t} & =\delta_{t} \bar{M}_{t} d W_{t}
\end{aligned}
$$

and if we differentiate these conditions we obtain the following system of equations

$$
\begin{aligned}
& \left(\alpha_{t}^{i} u_{c c}\left(t, y^{*^{*}}\right)+\frac{1}{2} \beta_{t}^{i 2} u_{c c c}\left(t, y^{*^{i}}\right)+\delta_{t}^{i} \beta_{t}^{i} u_{c c}\left(t, y^{*^{i}}\right)\right) M_{t}^{i}= \\
& \left(a_{t}^{i} u_{c c}\left(t, \bar{y}^{i}\right)+\frac{1}{2} b_{t}^{i 2} u_{c c c}\left(t, \bar{y}^{i}\right)+\delta_{t} b_{t}^{i} u_{c c}\left(t, \bar{y}^{i}\right)\right) \bar{M}_{t} \\
& \left(\delta_{t}^{i} u_{c}\left(t, y^{*^{*}}\right)+\beta_{t}^{i} u_{c c}\left(t, y^{*^{i}}\right)\right) M_{t}^{i}=\left(\delta_{t} u_{c}\left(t, \bar{y}^{i}\right)+b_{t}^{i} u_{c c}\left(t, \bar{y}^{i}\right)\right) \bar{M} \\
& \sum_{i} a_{t}^{i}=\bar{r}_{t} \sum_{i} \alpha_{t}^{i}+\rho_{t} \sum_{i} y^{*^{i}} \\
& \sum_{i} b_{t}^{i}=\bar{r}_{t} \sum_{i} \beta_{t}^{i}
\end{aligned}
$$


with the initial conditions

$$
\begin{aligned}
& \bar{r}_{0}=1 \\
& \bar{y}_{0}^{i}=y_{0}^{*^{i}} \\
& \bar{M}_{0}=1
\end{aligned}
$$

Proposition 3.1. There exists a unique solution $\left(\left(a_{t}^{i}\right),\left(b_{t}^{i}\right), \delta_{t}, \rho_{t}\right)$ to the system of equations 3.2.

This solution depends on $\left(\left(y^{*^{i}}\right),\left(M_{t}^{i}\right),\left(\bar{y}^{i}\right),(\bar{M}),\left(\alpha_{t}^{i}\right),\left(\beta_{t}^{i}\right),\left(\delta_{t}^{i}\right), \bar{r}_{t}\right)$. If it is regular enough in order to ensure that the stochastic differential equations

$$
\begin{aligned}
d \bar{y}_{t}^{i} & =a_{t}^{i} \bar{y}_{t}^{i} d t+b_{t}^{i} \bar{y}_{t}^{i} d W_{t}, \quad \bar{y}_{0}^{i}=y_{0}^{*^{i}} \\
d \bar{M}_{t} & =\delta_{t} \bar{M}_{t} d W_{t}, \quad \bar{M}_{0}=1 \\
d \bar{r}_{t} & =\rho_{t} \bar{r}_{t} d t, \quad \bar{r}_{0}=1
\end{aligned}
$$

admit a solution, then we have

$$
\begin{aligned}
\sum_{i=1}^{N} \bar{y}^{i} & =\bar{r} e^{*} \\
M^{i} u_{i}^{\prime}\left(\cdot, y^{*^{i}}\right) & =\bar{M} u_{i}^{\prime}\left(\cdot, \bar{y}^{i}\right)
\end{aligned}
$$

As in the discrete time framework, it is possible, in the HARA utility setting, to characterize more precisely these solutions.

Proposition 3.2. If we suppose that each utility function is such that $-\frac{u_{i}^{\prime}(t, x)}{u_{i}^{\prime \prime}(t, x)}=$ $\theta_{i}+\eta x$, the density $\bar{M}$ and the adjustment process $\bar{r}$ are given by

1. when $\eta \neq 0$,

$$
\begin{aligned}
& \bar{M} \text { is a martingale } \\
\bar{r}_{0}= & M_{0}=1 \\
\bar{M}_{t}= & \left(\frac{\bar{\theta}+\eta \bar{r}_{t} e_{t}}{\bar{\theta}+\eta e_{t}}\right)^{1 / \eta}\left[\sum_{i=1}^{N} \gamma_{i}\left(M_{t}^{i}\right)^{\eta}\right]^{1 / \eta}
\end{aligned}
$$

with $\gamma_{i}=\frac{\lambda_{i}^{-\eta}}{\sum_{j} \lambda_{j}^{-\eta}}$. 
2. when $\eta=0$, by

$$
\begin{aligned}
\bar{M} \text { is a martingale } \\
\bar{r}_{0}=\overline{M_{0}}=1 \\
\overline{M_{t}}=\prod_{i=1}^{N}\left(M_{t}^{i}\right)^{\theta_{i} / \bar{\theta}} \exp \frac{\left(\overline{r_{t}}-1\right) e_{t}}{\bar{\theta}}
\end{aligned}
$$

3. If $\bar{\theta} \equiv \sum_{i=1}^{N} \theta_{i}=0$ and $\eta \neq 0$, then we obtain a simple construction algorithm

$$
\begin{aligned}
& \bar{M} \text { is a martingale } \\
\bar{r}_{0}= & \overline{M_{0}}=1 \\
\left(\overline{M_{t}}\right)^{\eta}= & \overline{r_{t}}\left[\sum_{i=1}^{N} \gamma_{i}\left(M_{t}^{i}\right)^{\eta}\right]
\end{aligned}
$$

and the adjustment process $\bar{r}$ satisfies

$$
\begin{aligned}
& \bar{r} \text { is nondecreasing if } \eta<1 \\
& \bar{r} \text { is nonincreasing if } \eta>1 .
\end{aligned}
$$

In particular, as in the discrete time setting, we find that beliefs heterogeneity leads to an equivalent equilibrium with increased aggregate endowment if and only if the investor is cautious, i.e. if the cautiousness parameter $\eta$ is smaller than one.

\subsection{Comonotonicity property and the second requirement}

A desirable property of the "equivalent equilibrium" is that each investor's observed (or initial) demand be larger than (resp. equal to, less than) his demand in the "equivalent equilibrium" if and only if he attaches a subjective probability that is larger than (resp. equal to, less than) the aggregate common probability, i.e.

$$
y_{t}^{*^{i}} \geq \bar{y}_{t}^{i} \text { if and only if } M_{t}^{i} \geq \bar{M}_{t}
$$

and

$$
y_{t}^{*^{i}} \leq \bar{y}_{t}^{i} \text { if and only if } M_{t}^{i} \leq \bar{M}_{t}
$$

that we shall refer to as the comonotonicity property (at date $t$ ). We want in this subsection to analyze the links between this comonotonicity property and the 
second invariance requirement of our aggregation procedure, i.e. the invariance of individual marginal valuations.

It is easy to see that, due to our second invariance requirement, the comonotonicity property is automatically satisfied by our aggregation procedure. Indeed, as seen previously, we have for all $i=1, \ldots, N$ and for all $t=0, \ldots, T$,

$$
M_{t}^{i} u_{i}^{\prime}\left(t, y_{t}^{*^{i}}\right)=\bar{M}_{t} u_{i}^{\prime}\left(t, \bar{y}_{t}^{i}\right)
$$

and, since $u_{i}^{\prime}(t, \cdot)$ is decreasing, the comonotonicity property follows immediately at any date $t=0, \ldots, T$.

Conversely, let us study to which extent the comonotonicity property as well as the first requirement imply our second requirement. By the first requirement, we must have for all $i=1, \ldots, N$

$$
\frac{M_{t}^{i}}{\bar{M}_{t}}=\frac{\lambda_{i}}{\zeta_{i}} \frac{u_{i}^{\prime}\left(t, \bar{y}_{t}^{i}\right)}{u_{i}^{\prime}\left(t, y_{t}^{*^{i}}\right)},
$$

where $\lambda_{i}$ is as above the Lagrange multiplier in the initial equilibrium, and $\zeta_{i}$ is the Lagrange multiplier in the "equivalent equilibrium." The second requirement is then equivalent to the condition that $\frac{\lambda_{i}}{\zeta_{i}}=1$ for all $i=1, \ldots, N$. Imposing directly the comonotonicity property, instead of the second requirement, leads to

$$
\frac{M_{t}^{i}}{\bar{M}_{t}} 1_{\left\{\frac{M_{t}^{i}}{\bar{M}_{t}} \leq 1\right\}} \leq \frac{\lambda_{i}}{\zeta_{i}} \leq \frac{M_{t}^{i}}{\bar{M}_{t}} 1_{\left\{\frac{M_{t}^{i}}{\bar{M}_{t}} \geq 1\right\}} .
$$

If we require the comonotonicity property to be satisfied from date 0 , then it is immediate that it implies our second requirement: indeed, since $M_{0}^{i}=\bar{M}_{0}=1$, we get by $(3.3)$ at $t=0$, that $\frac{\lambda_{i}}{\zeta_{i}}=1$ for all $i=1, \ldots, N$.

If we only require the comonotonicity property from date $t=1$, then it is immediate that the following condition $(C 1)$ implies the second requirement.

Condition $(C 1)$ : For all $i \in\{1, \ldots, N\}$, for all $\varepsilon \in \mathbb{R}_{+}^{*}$, there exists some $t \in$ $\{1, \ldots, T\}$ for which

$$
\left.\left.P\left\{\frac{M_{t}^{i}}{\bar{M}_{t}} \in\right] 1-\varepsilon, 1\right]\right\} \times P\left\{\frac{M_{t}^{i}}{\bar{M}_{t}} \in[1,1+\varepsilon[\}>0 .\right.
$$

Notice that condition $(C 1)$ can be interpreted as a closeness condition between $\bar{M}$ and the $M^{i}$ s. 
We introduce the following condition.

Condition $(C 2)$ : For all $i \in\{1, \ldots, N\}$, there exists some $t \in\{1, \ldots, T\}$ for which (a) The vector $\left(M_{t}^{1}, \ldots, M_{t}^{N}, e_{t}^{*}\right)$ admits a positive density with respect to the Lebesgue measure on $\left(\mathbb{R}_{+}\right)^{N} \times[\underline{e}, \bar{e}]$.

(b) The random variable $\bar{M}_{t}$ can be written in the form $\bar{M}_{t}=g\left(M_{t}^{1}, \ldots, M_{t}^{n}, e_{t}^{*}\right)$ for some function $g$ of class $C^{1}$ such that $x_{i} \frac{\partial g}{\partial x_{i}}-g \neq 0$.

Notice that condition $(C 2)(b)$ is satisfied if $g$ is concave and nonnegative.

We obtain the following result:

Proposition 3.3. $\quad$ 1. If the aggregation procedure satisfies the first requirement and the comonotonicity property at all dates $t=0, \ldots, T$, then the "equivalent equilibrium" satisfies the second requirement.

2. If the aggregation procedure satisfies the first requirement, the comonotonicity property at dates $t=1, \ldots, T$ as well as $(C 2)$, then the "equivalent equilibrium" satisfies the second requirement.

\subsection{Other possible aggregation procedures}

We have considered so far extensions of Calvet et al. (2002). More precisely, knowing that a true aggregation of individual beliefs leaving all equilibrium characteristics invariant is impossible, we have proposed aggregation procedures that authorize an adjustment of the market portfolio. Another possible aggregation procedure consists in leaving the market portfolio invariant and in authorizing the introduction of a discount factor on the utility functions. We show in Jouini-Napp (2003), in continuous time, that given an equilibrium price process $q^{*}$ relative to the beliefs $\left(M^{i}\right)$, and the endowment processes $\left(e^{i}\right)$ with $\sum_{i=1}^{N} e^{i}=e^{*}$, there exists a positive martingale process $\bar{M}$ with $\bar{M}_{0}=1$, and a finite variation positive process $B \equiv \exp \int_{0}^{*} \mu(s) d s$ such that, with the notations of Section 2,

$$
\bar{M}_{t} B_{t} u_{\lambda}^{\prime}\left(t, e_{t}^{*}\right)=q_{t}^{*} .
$$

The adjustment process $B$ measures then the aggregation bias induced by the heterogeneity of individual beliefs and leads to a (possibly negative) discount of utility from future consumption through the "discount rate" $(-\mu)$. It is shown in Jouini-Napp (2003) that the consensus belief $\bar{M}$ is given by some weighted average of the individual beliefs, the weights being given by the individual risk tolerances, and that the process $B$ (or $\mu$ ) is directly related to the weighted variance (with the same weights) of the individual beliefs. 


\section{Appendix}

Proof of Proposition 2.1 Since $\left(q^{*},\left(y^{*^{i}}\right)\right)$ is an equilibrium relative to the beliefs $\left(Q^{i}\right)$, and the endowment processes $e^{i}$ with $\sum_{i=1}^{N} e^{i}=e^{*}$, we know that for all $t=0, \ldots, T$,

$$
\sum_{i=1}^{N} y_{t}^{*^{i}}=\sum_{i=1}^{N} e_{t}^{i}=e_{t}^{*}
$$

and that there exist positive Lagrange multipliers $\left(\lambda_{i}\right)$ such that for all $i=$ $1, \ldots, N$, for all $t=0, \ldots, T$,

$$
M_{t}^{i} u_{i}^{\prime}\left(t, y_{t}^{*^{i}}\right)=\lambda_{i} q_{t}^{*}
$$

where $M_{t}^{i} \equiv E\left[\frac{d Q^{i}}{d P} \mid F_{t}\right]$. For a given bounded and nonnegative predictable process $r$, we consider the maximization problem

$$
\left(\mathcal{P}^{r}\right): \max \sum_{i=1}^{N} \frac{1}{\lambda_{i}} U_{i}\left(y^{i}\right) \text { under the constraint } \sum_{i=1}^{N} y^{i} \leq r e^{*},
$$

where $U_{i}(c)=E\left[\sum_{t=0}^{T} u_{i}\left(t, c_{t}\right)\right]$.

If we denote by $\left(y^{i,(r)}\right)$ the solution of the maximization program $\left(\mathcal{P}^{r}\right)$, then the positive process $\left(\frac{1}{\lambda_{i}} u_{i}^{\prime}\left(t, y_{t}^{i,(r)}\right)\right)_{t=0}^{T}$ is independent from $i$ and we denote it by $p^{(r)}$. Let $M^{(r)} \equiv \frac{q^{*}}{p^{(r)}}$, where by convention, $M^{(r)}=0$ on $\left\{p^{(r)}=\infty\right\}$. We then have for all $i$, for all $t=0, \ldots, T$

$$
M_{t}^{(r)}=M_{t}^{i} \frac{u_{i}^{\prime}\left(t, y_{t}^{*^{i}}\right)}{u_{i}^{\prime}\left(t, y_{t}^{i,(r)}\right)}
$$

Notice that for all $t$, the random variables $y_{t}^{i,(r)}\left(\operatorname{resp} . M_{t}^{(r)}\right)$ depend on the process $r$ only through $r_{t}$ and can therefore be denoted by $y_{t}^{i,\left(r_{t}\right)}\left(\operatorname{resp} . M_{t}^{\left(r_{t}\right)}\right)$. We want to find a positive and bounded predictable process $\bar{r}$ such that $M_{0}^{(\bar{r})}=1$ and $M^{(\bar{r})}$ is a martingale. Let us construct such a process by induction on $t$.

For $t=0$, we take $\overline{r_{0}}=1$, and we get $y_{0}^{i,\left(\overline{r_{0}}\right)}=y_{0}^{*^{i}}$ and $M_{0}^{\left(\overline{r_{0}}\right)}=1$. Let us suppose that we have obtained the positive and bounded predictable process $\left(\bar{r}_{s}\right)_{s=0}^{t-1}$, hence 
the positive process $\left(M_{s}^{(\bar{r})}\right)_{s=0}^{t-1}$ and let us construct $\bar{r}_{t}$ such that $E\left[M_{t}^{\left(\overline{r_{t}}\right)} \mid F_{t-1}\right]=$ $M_{t-1}^{\left(\overline{r_{t-1}}\right)}$. We introduce the function $\Phi: r_{t} \mapsto E\left[M_{t}^{\left(r_{t}\right)} \mid F_{t-1}\right]-M_{t-1}^{\left(\overline{r_{t-1}}\right)}$ and we want to prove that there exists an $F_{t-1}$-measurable positive and bounded random variable $\overline{r_{t}}$ such that $\Phi\left(\overline{r_{t}}\right)=0$ (almost surely).

We first show that there exists a positive constant $R$ such that any nonnegative and bounded $F_{t-1}$-measurable random variable $a$ satisfies $\Phi(a)>0$ on $\{a>R\}$. Notice that since $u_{i}^{\prime}\left(t, y_{t}^{i,(a)}\right)=\frac{\lambda_{i} q_{t}^{*}}{M_{t}^{(a)}}$, we have $\frac{q_{t}^{*}}{M_{t}^{(a)}}=h_{t}\left(a e_{t}^{*}\right)$ with $h_{t}^{-1}(x)=$ $\sum_{i=1}^{N}\left(u_{i}^{\prime}\right)^{-1}\left(t, \lambda_{i} x\right)$. We have

$$
\Phi(a)=E\left[\frac{M_{t}^{i} u_{i}^{\prime}\left(t, y_{t}^{*^{i}}\right)}{\lambda_{i} h_{t}\left(a e_{t}^{*}\right)} \mid F_{t-1}\right]-M_{t-1}^{\left(\overline{r_{t-1}}\right)}
$$

hence

$$
\begin{aligned}
\Phi(a) 1_{\{a>R\}} & >E\left[\frac{M_{t}^{i} u_{i}^{\prime}(t, \bar{e})}{\lambda_{i} h_{t}(\underline{e} R)} 1_{\{a>R\}} \mid F_{t-1}\right]-M_{t-1}^{\left(\overline{r_{t-1}}\right)} 1_{\{a>R\}} \\
& >\left[M_{t-1}^{i} \frac{u_{i}^{\prime}(t, \bar{e})}{\lambda_{i} h_{t}(\underline{e} R)}-M_{t-1}^{\left(\overline{r_{t-1}}\right)}\right] 1_{\{a>R\}}
\end{aligned}
$$

so that the existence of $R$ such that $\Phi(a)>0$ on $\{a>R\}$ is given by the existence of a positive constant $A$ such that $\inf _{i} \frac{M_{t-1}^{\left(\bar{T}_{t-1}\right)}}{M_{t-1}^{i}}<A$. Now,

$$
\frac{M_{t-1}^{\left(\overline{r_{t-1}}\right)}}{M_{t-1}^{i}}=\frac{u_{i}^{\prime}\left(t-1, y_{t-1}^{*^{i}}\right)}{u_{i}^{\prime}\left(t-1, y_{t}^{i,\left(\overline{r_{t-1}}\right)}\right)}
$$

and

$$
\inf _{i} \frac{\left.M_{t-1}^{(\bar{r}-1}\right)}{M_{t-1}^{i}}<\sup _{i} \frac{u_{i}^{\prime}\left(t-1, \frac{e}{N}\right)}{u_{i}^{\prime}\left(t-1, R_{t-1} \bar{e}\right)}
$$

where $R_{t-1}$ denotes the upper bound for $\overline{r_{t-1}}$.

Let us consider the set $\Gamma$ of nonnegative and bounded $F_{t-1}$-measurable random variables $r_{\gamma}$ such that $\Phi\left(r_{\gamma}\right) \leq 0$. The set $\Gamma$ contains 0 and we have just proved that any $r_{\gamma}$ in $\Gamma$ is bounded by $R$. Furthermore, it is easy to check that, for all $F_{t-1}$-nonnegative and bounded measurable random variables $r_{1}$ and $r_{2}$, and any 
event $B \in F_{t-1}, \Phi\left(r_{1} 1_{B}+r_{2} 1_{B^{c}}\right)=\Phi\left(r_{1}\right) 1_{B}+\Phi\left(r_{2}\right) 1_{B^{c}}$ and the monotonicity of $M^{(r)}$ with respect to $r$ leads to $\Phi\left(r_{1} \vee r_{2}\right)=\Phi\left(r_{1}\right) \vee \Phi\left(r_{2}\right)$.

Let $r^{*} \equiv e s s \sup _{r_{\gamma} \in \Gamma} r_{\gamma}$. The family being directed upward, we know that there exists $\left(r_{\gamma_{k}}\right)$ in $\Gamma$ such that $r^{*}=\lim _{k \rightarrow \infty} \nearrow r_{\gamma_{k}}$. We prove now that $\Phi\left(r^{*}\right)=0$.

It is immediate that $r^{*}$ is nonnegative, bounded and predictable. Besides, it is easy to see that, when $k \rightarrow \infty$, then $M^{\left(r_{\gamma_{k}}\right)} \nearrow M^{\left(r^{*}\right)}$, and since for all $k, \Phi\left(r_{\gamma_{k}}\right) \leq 0$, we get by the Beppo-Levi Theorem that $\Phi\left(r^{*}\right) \leq 0$. It remains to show that for all $(\varepsilon, b) \in\left(\mathbb{R}_{+}^{*}\right)^{2}$, we have $P\left(\Omega_{\varepsilon, b}\right)=0$, where $\Omega_{\varepsilon, b} \equiv$ $\left\{\Phi\left(r^{*}\right)<-\varepsilon, E_{t-1}\left[q_{t}^{*}\right] \leq b\right\} \in F_{t-1}$. We introduce $\widehat{r}=r^{*}+\alpha 1_{\Omega_{\varepsilon, b}}$ for $\alpha \in \mathbb{R}_{+}^{*}$. We get $\Phi(\widehat{r})=\Phi\left(r^{*}\right)+\left[\Phi\left(r^{*}+\alpha\right)-\Phi\left(r^{*}\right)\right] 1_{\Omega_{\varepsilon, b}}$. We shall prove that for some positive $\alpha,\left|\Phi\left(r^{*}+\alpha\right)-\Phi\left(r^{*}\right)\right|<\varepsilon$ on $\Omega_{\varepsilon, b}$. This would lead to $\Phi(\widehat{r}) \leq 0$, hence $P\left(\Omega_{\varepsilon, b}\right)=0$ and $\Phi\left(r^{*}\right)=0$.

Now,

$$
\begin{aligned}
\left|\Phi\left(r^{*}+\alpha\right)-\Phi\left(r^{*}\right)\right| \mathbf{1}_{\Omega_{\varepsilon, b}} & =\left|E_{t-1}\left[q_{t}^{*}\left(\frac{1}{h_{t}\left(\left(r^{*}+\alpha\right) e_{t}^{*}\right)}-\frac{1}{h_{t}\left(r^{*} e_{t}^{*}\right)}\right)\right]\right| \mathbf{1}_{\Omega_{\varepsilon, b}} \\
& \leq b \sup _{\substack{|x-y| \leq \alpha \bar{e} \\
x, y \in[0, R \bar{e}]}}|\Psi(x)-\Psi(y)| \mathbf{1}_{\Omega_{\varepsilon, b}}
\end{aligned}
$$

for $\Psi \equiv \frac{1}{h_{t}}$ and then $\left|\Phi\left(r^{*}+\alpha\right)-\Phi\left(r^{*}\right)\right|<\varepsilon$ on $\Omega_{\varepsilon, b}$ for $\alpha$ small enough.

Taking $\overline{r_{t}}=r^{*}$ completes the construction by induction of the process $\bar{r}$.

As far as uniqueness is concerned, it is first easy to see that for any positive and bounded process $\bar{r}$, there exists a unique $\left(\bar{M},\left(\bar{y}^{i}\right),\left(\bar{e}^{i}\right)\right)=\left(M^{(\bar{r})}, y^{i,(\bar{r})}, e^{i}-y^{*^{i}}+\bar{y}^{i}\right)$ satisfying requirements 1 . and 2 . of the Theorem. The uniqueness of $\bar{r}$ is immediate by monotonicity of $\Phi$.

Proof of Proposition 2.2 Similar to the proof of the existence of a representative agent in the homogeneous beliefs setting.

\section{Proof of Proposition 2.3}

1) We know that

$$
\frac{1}{\lambda_{i}} M_{t}^{i} u_{i}^{\prime}\left(t, y_{t}^{*^{i}}\right) \underset{(1)}{=} \bar{M}_{t} u_{\lambda}^{\prime}\left(t, \bar{r}_{t} e_{t}^{*}\right) \underset{(2)}{=} \frac{1}{\lambda_{i}} \bar{M}_{t} u_{i}^{\prime}\left(t, \bar{y}_{t}^{i}\right)
$$

We get from the second equality that $u_{\lambda}^{\prime}\left(t, \bar{r}_{t} e_{t}^{*}\right)=\frac{1}{\lambda_{i}} u_{i}^{\prime}\left(t, \bar{y}_{t}^{i}\right)$ so that, for $\eta>0,\left[u_{i}^{\prime}\left(t, \bar{y}_{t}^{i}\right)\right]^{-\eta}=\left[\lambda_{i} u_{\lambda}^{\prime}\left(t, \bar{r}_{t} e_{t}^{*}\right)\right]^{-\eta}$. Since $u_{i}^{\prime}(t, x)=\left(\theta_{i}+\eta x\right)^{-1 / \eta}$, this leads to $\theta_{i}+\eta y_{t}^{i,(r)}=\left[\lambda_{i} u_{\lambda}^{\prime}\left(t, \bar{r}_{t} e_{t}^{*}\right)\right]^{-\eta}$, hence $u_{\lambda}^{\prime}\left(t, \bar{r}_{t} e_{t}^{*}\right)^{-\eta}=\frac{\bar{\theta}+\eta \bar{r}_{t} e_{t}^{*}}{\sum_{i=1}^{N} \lambda_{i}^{-\eta}}$. From the first 
equality, we get that $\left[u_{i}^{\prime}\left(t, y_{t}^{*^{i}}\right)\right]^{-\eta}=\left[\lambda_{i} \frac{\bar{M}_{t}}{M_{t}^{i}} u_{\lambda}^{\prime}\left(t, \bar{r}_{t} e_{t}^{*}\right)\right]^{-\eta}=\theta_{i}+\eta y_{t}^{*^{i}}$, hence $\bar{\theta}+$ $\eta e_{t}^{*}=\bar{M}_{t}^{-\eta} u_{\lambda}^{\prime}\left(t, \bar{r}_{t} e_{t}^{*}\right)^{-\eta} \sum_{i=1}^{N}\left(\frac{\lambda_{i}}{M_{t}^{i}}\right)^{-\eta}$, and $\bar{M}_{t}=\left(\frac{\bar{\theta}+\eta \bar{r}_{t} e_{t}^{*}}{\bar{\theta}+\eta e_{t}}\right)^{1 / \eta}\left[\sum_{i=1}^{N} \gamma_{i}\left(M_{t}^{i}\right)^{\eta}\right]^{1 / \eta}$.

If $\eta=0$, we have $u_{i}^{\prime}(t, x)=\exp -\frac{x}{\theta_{i}}$ and adopting the same approach, using the second equality $u_{\lambda}^{\prime}\left(t, \bar{r}_{t} e_{t}^{*}\right)=\exp -\frac{\bar{r}_{t} e_{t}^{*}}{\bar{\theta}} \exp -\frac{1}{\bar{\theta}} \sum_{i=1}^{N} \theta_{i} \ln \lambda_{i}$. From the first equality, we get that $\exp -\frac{y_{t}^{*^{i}}}{\theta_{i}}=\lambda_{i} \frac{\bar{M}_{t}}{M_{t}^{i}} \exp -\frac{\bar{r}_{t} e_{t}^{*}}{\bar{\theta}} \exp -\frac{1}{\bar{\theta}} \sum_{i=1}^{N} \theta_{i} \ln \lambda_{i}$, and then $\bar{M}_{t}=\prod_{i=1}^{N}\left(M^{i}\right)^{\theta_{i} / \bar{\theta}} \exp \frac{\left(\bar{r}_{t}-1\right) e_{t}^{*}}{\bar{\theta}}$.

2) Since $\bar{M}_{t}=\left(\frac{\bar{\theta}+\eta \bar{r}_{t} e_{t}^{*}}{\bar{\theta}+\eta e_{t}^{*}}\right)^{1 / \eta}\left[\sum_{i=1}^{N} \gamma_{i}\left(M_{t}^{i}\right)^{\eta}\right]^{1 / \eta}$, we have

$$
E_{t-1}\left[\bar{M}_{t}\right] \mathbf{1}_{\bar{r}_{t}<1}<E_{t-1}\left[\left[\sum_{i=1}^{N} \gamma_{i}\left(M_{t}^{i}\right)^{\eta}\right]^{1 / \eta}\right] \mathbf{1}_{\bar{r}_{t}<1} .
$$

Now, for $0<\eta<1$, we get by Minkovski's inequality $E_{t-1}\left[\left[\sum_{i=1}^{N} \gamma_{i}\left(M_{t}^{i}\right)^{\eta}\right]^{1 / \eta}\right] \leq$ $\left[\sum_{i=1}^{N} \gamma_{i}\left(M_{t-1}^{i}\right)^{\eta}\right]^{1 / \eta}$, hence $E_{t-1}\left[\bar{M}_{t}\right] \mathbf{1}_{\bar{r}_{t}<1}<\bar{M}_{t-1}\left(\frac{\bar{\theta}+\eta \bar{r}_{t-1} e_{t-1}^{*}}{\bar{\theta}+\eta e_{t-1}^{*}}\right)^{-1 / \eta} \mathbf{1}_{\bar{r}_{t}<1}$, and

$$
\left(\frac{\bar{\theta}+\eta \bar{r}_{t-1} e_{t-1}^{*}}{\bar{\theta}+\eta e_{t-1}^{*}}\right) \mathbf{1}_{\bar{r}_{t}<1}<\mathbf{1}_{\bar{r}_{t}<1} .
$$

This leads to $\left\{\bar{r}_{t}<1\right\} \subset\left\{\bar{r}_{t-1}<1\right\}$. Since $\bar{r}_{0}=1$, we have $\bar{r} \geq 1$. The case $\eta>1$ can be treated similarly. For $\eta=1$, it is clear that $\bar{r}=1$ and $\bar{M}=\sum_{i=1}^{N} \gamma_{i} M^{i}$ is the solution of our equations.

For $\eta=0, E_{t-1}\left[\bar{M}_{t}\right] \mathbf{1}_{\bar{r}_{t}<1} \leq E_{t-1}\left[\prod_{i}\left(M_{t}^{i}\right)^{\theta_{i} / \bar{\theta}}\right] \mathbf{1}_{\bar{r}_{t}<1} \leq \prod_{i=1}^{N}\left(\bar{M}_{t-1}^{i}\right)^{\theta_{i} / \bar{\theta}}$ and then $\mathbf{1}_{\bar{r}_{t}<1} \leq \exp -\frac{\left(\bar{r}_{t-1}-1\right) e_{t-1}^{*}}{\bar{\theta}} \mathbf{1}_{\bar{r}_{t}<1}$ and as previously $\left\{\bar{r}_{t}<1\right\} \subset\left\{\bar{r}_{t-1}<1\right\}$.

3) In the case $\bar{\theta} \equiv \sum_{i=1}^{N} \theta_{i}=0$, it is immediate that $\left(\bar{M}_{t}\right)^{\eta}=\bar{r}_{t}\left[\sum_{i=1}^{N} \gamma_{i}\left(M_{t}^{i}\right)^{\eta}\right]$ hence $E_{t-1}\left[\bar{M}_{t}\right]<\bar{r}_{t}^{1 / \eta} E_{t-1}\left[\left[\sum_{i=1}^{N} \gamma_{i}\left(M_{t}^{i}\right)^{\eta}\right]^{1 / \eta}\right]$. Now, for $\eta>1$, we get the following Minkovski-like inequality $E_{t-1}\left[\left[\sum_{i=1}^{N} \gamma_{i}\left(M_{t}^{i}\right)^{\eta}\right]^{1 / \eta}\right] \geq\left[\sum_{i=1}^{N} \gamma_{i}\left(M_{t-1}^{i}\right)^{\eta}\right]^{1 / \eta}$, hence $\bar{M}_{t-1} \geq \bar{r}_{t}^{1 / \eta}\left[\sum_{i=1}^{N} \gamma_{i}\left(M_{t-1}^{i}\right)^{\eta}\right]^{1 / \eta}$, and $\bar{M}_{t-1} \geq\left(\frac{\bar{r}_{t}}{\bar{r}_{t-1}}\right)^{1 / \eta} \bar{M}_{t-1}$ or equivalently $\frac{\bar{r}_{t}}{\bar{r}_{t-1}} \leq 1$. The case $\eta>1$ can be treated similarly. 
Proof of Proposition 3.1 The second equation in the system 3.2 gives us

$$
\frac{\left(\delta_{t}^{i} u_{c}\left(t, y^{*^{i}}\right)+\beta_{t}^{i} u_{c c}\left(t, y^{*^{i}}\right)\right) M_{t}^{i}}{u_{c c}\left(t, \bar{y}^{i}\right) \bar{M}}=\delta_{t} \frac{u_{c}\left(t, \bar{y}^{i}\right)}{u_{c c}\left(t, \bar{y}^{i}\right)}+b_{t}^{i}
$$

and we deduce from the fourth equation in the same system that

$$
\delta_{t}=\frac{\sum_{i=1}^{N} \frac{\left(\delta_{t}^{i} u_{c}\left(t, y^{*^{i}}\right)+\beta_{t}^{i} u_{c c}\left(t, y^{*^{i}}\right)\right) M_{t}^{i}}{u_{c c}\left(t, \bar{y}^{i}\right) \bar{M}}-\bar{r}_{t} \sum_{i=1}^{N} \beta_{t}^{i}}{\sum_{i=1}^{N} \frac{u_{c}\left(t, \bar{y}^{i}\right)}{u_{c c}\left(t, \bar{y}^{i}\right)}} .
$$

This gives $\delta_{t}$ and the $b^{i}$ 's.

The $a_{i}$ 's (resp. $\rho$ ) are then obtained from the first (resp. the third) equation in the system 3.2.

Proof of Proposition 3.2 The relations between $\bar{M}, \bar{r}$ and the $M^{i}$ 's are obtained exactly as in the discrete time framework. In the particular case where $\bar{\theta}=0$, we have

$$
\left(\overline{M_{t}}\right)^{\eta}=\overline{r_{t}}\left[\sum_{i=1}^{N} \gamma_{i}\left(M_{t}^{i}\right)^{\eta}\right]
$$

and if we differentiate both sides, we obtain

$$
\begin{aligned}
\frac{1}{2} \overline{\gamma_{t}} \eta(\eta-1) \sum_{i=1}^{N} \gamma_{i}\left(\delta_{t}^{i}\right)^{2}\left(M_{t}^{i}\right)^{\eta}+\bar{\rho}_{t} \bar{r}_{t} \sum_{i=1}^{N} \gamma_{i}\left(M_{t}^{i}\right)^{\eta} & =\frac{1}{2} \eta(\eta-1) \delta_{t}^{2} M_{t}^{\eta} \\
\overline{r_{t}} \eta \sum_{i=1}^{N} \gamma_{i} \delta_{t}^{i}\left(M_{t}^{i}\right)^{\eta} & =\eta \delta_{t} M_{t}^{\eta}
\end{aligned}
$$

or equivalently

$$
\begin{aligned}
\delta_{t} & =\sum_{i=1}^{N} \mu_{t}^{i} \delta_{t}^{i} \\
\bar{\rho}_{t} & =\frac{1}{2} \eta(\eta-1)\left[\left(\sum_{i=1}^{N} \mu_{t}^{i} \delta_{t}^{i}\right)^{2}-\sum_{i=1}^{N} \mu_{t}^{i}\left(\delta_{t}^{i}\right)^{2}\right]
\end{aligned}
$$

where for all $i, \mu_{t}^{i} \equiv \overline{r_{t}} \gamma_{i} \frac{\left(M_{t}^{i}\right)^{\eta}}{M_{t}^{\eta}}$ and satisfy $\sum_{i=1}^{N} \mu_{t}^{i}=1$ (by 4.2). 
Since $\left(\sum_{i=1}^{N} \mu_{t}^{i} \delta_{t}^{i}\right)^{2}-\sum_{i=1}^{N} \mu_{t}^{i}\left(\delta_{t}^{i}\right)^{2}$ is always nonpositive, $\bar{\rho}_{t}$ and $(\eta-1)$ have opposite signs and $\bar{r}$ is nondecreasing (resp. nonincreasing) for $\eta<1$ (resp. $\eta>1)$.

Proof of Proposition 3.3 It is easy to see that under condition $(C 2)$, the application

$$
h:\left(x_{1}, \ldots, x_{N}, y\right) \mapsto\left(x_{1}, \ldots x_{i-1}, \frac{x_{i}}{g\left(x_{1}, \ldots, x_{N}, y\right)}, x_{i+1}, \ldots, x_{N}, y\right)
$$

is a $C^{1}$-diffeomorphism from $\left.\left(\mathbb{R}_{+}^{*}\right)^{N} \times\right] \underline{e}, \bar{e}\left[\right.$ to an open subset $U$ of $\left.\left(\mathbb{R}_{+}^{*}\right)^{N} \times\right] \underline{e}, \bar{e}[$. Then the random vector $\left(M_{t}^{1}, \ldots, M_{t}^{i-1}, \frac{M_{t}^{i}}{M_{t}}, M_{t}^{i+1}, \ldots, M_{t}^{N}, e_{t}^{*}\right)$ admits a density with respect to the Lebesgue measure on $\left(\mathbb{R}_{+}\right)^{N} \times[\underline{e}, \bar{e}]$, which is positive on $U$. Furthermore, since $E\left[\bar{M}_{t}\right]=E\left[M_{t}^{i}\right]=1$, it is impossible to have $g\left(x_{1}, \ldots, x_{N}, y\right)<x_{i}$ for all $\left(x_{1}, \ldots, x_{N}, y\right)$, or $g\left(x_{1}, \ldots, x_{N}, y\right)>x_{i}$ for all $\left(x_{1}, \ldots, x_{N}, y\right)$. Since $\operatorname{Im} U$ is connected, $\operatorname{Pr} o j_{i}(\operatorname{Im} U)$ is also connected, where $\operatorname{Pr} o j_{i}$ denotes the projection on the $i$-th coordinate, hence there exists $\left(\widehat{x}_{1}, \ldots, \widehat{x}_{N}, \widehat{y}\right)$ such that $g\left(\widehat{x}_{1}, \ldots, \widehat{x}_{N}, \widehat{y}\right)=\widehat{x}_{i}$. Since $U$ is open, there exists a neighborhood of $\left(\widehat{x}_{1}, \ldots, \widehat{x}_{i-1}, 1, \widehat{x}_{i+1}, \ldots, \widehat{x}_{N}, \widehat{y}\right)$ in $U$, which leads to

$$
\left.\left.P\left\{\frac{M_{t}^{i}}{\bar{M}_{t}} \in\right] 1-\varepsilon, 1\right]\right\} \times P\left\{\frac{M_{t}^{i}}{\bar{M}_{t}} \in[1,1+\varepsilon[\}>0 .\right.
$$

\section{References}

[1] Basak, S. and D. Cuoco, 1998. An equilibrium model with restricted stock market participation. Review of Financial Studies, 11, 309-341.

[2] Calvet, L., Grandmont, J.-M., and I. Lemaire, 2002. Aggregation of Heterogenous Beliefs and Asset Pricing in Complete Financial Markets. Working Paper.

[3] Cuoco, D. and H. He, 1994. Dynamic equilibrium in infinite-dimensional economies with incomplete financial markets. Mimeo, University of Pennsylvania.

[4] Jouini, E., and C. Napp, 2003. Consensus Consumer and Intertemporal Asset Pricing with Heterogeneous Beliefs. Working Paper. 
[5] Rubinstein, M., 1974. An aggregation theorem for securities markets. Journal of Financial Economics, 1, 225-244.

[6] Rubinstein, M., 1976. The Strong Case for the Generalized Logarithmic Utility Model as the Premier Model of Financial Markets. Journal of Finance, 31, 551571.

[7] Zapatero, F., 1998. Effects of Financial Innovations on Market volatility when Beliefs are Heterogeneous. Journal of Economics, Dynamics and Control, 22, 597-626. 